\title{
Multiple scattering calculations of relativistic electron energy loss spectra
}

\author{
K. Jorissen, ${ }^{1}$ J. J. Rehr, ${ }^{1, *}$ and J. Verbeeck ${ }^{2}$ \\ ${ }^{1}$ Department of Physics, University of Washington, Seattle, Washington 98195, USA \\ ${ }^{2}$ EMAT, University of Antwerp, Groenenborgerlaan 171, B2020, Antwerp, Belgium
}

(Received 26 October 2009; revised manuscript received 30 March 2010; published 13 April 2010)

\begin{abstract}
A generalization of the real-space Green's-function approach is presented for ab initio calculations of relativistic electron energy loss spectra (EELS) which are particularly important in anisotropic materials. The approach incorporates relativistic effects in terms of the transition tensor within the dipole-selection rule. In particular, the method accounts for relativistic corrections to the magic angle in orientation resolved EELS experiments. The approach is validated by a study of the graphite $\mathrm{C} K$ edge, for which we present an accurate magic angle measurement consistent with the predicted value.
\end{abstract}

DOI: 10.1103/PhysRevB.81.155108

PACS number(s): 79.20.Uv, 71.15.Qe, 71.15.Rf

\section{INTRODUCTION}

Electron energy loss spectroscopy (EELS) measures the energy loss of a beam of high-energy electrons, typically of order $100 \mathrm{keV}$, propagated through a sample, e.g., in an electron microscope. ${ }^{1}$ The energy loss spectrum is defined as the fraction of electrons that have lost a given amount of energy by interacting inelastically with the sample. From the EELS spectrum, one can obtain structural, chemical, and electronic information analogous to that in X-ray absorption spectroscopy (XAS). The XAS spectrum encompasses both extended $\mathrm{X}$-ray absorption fine structure about $50 \mathrm{eV}$ above an absorption edge and x-ray absorption near-edge structure below that. We focus here on the ELNES (energy loss near-edge structure) edges in the EELS, corresponding to inelastic losses through the excitation of an electron from a deep core level into unoccupied states. Because EELS is an absorption technique and the initial core-level states are sharply defined in energy, such core loss signals reflect the electronic structure of unoccupied electron states. In particular, apart from a smoothly varying background cross-section factor, the shape of the ionization edge is roughly an image of the unoccupied and local angular momentum projected density of electron states (lDOS). Due to approximate dipole-selection rules, the observed spectrum corresponds to a decomposition of the DOS according into various angular momentum $l$ channels. Moreover, the possibility of tilting the specimen with respect to the electron beam at fixed scattering angle allows one to investigate the anisotropy in the local unoccupied DOS (Ref. 2) because the momentum transfer or scattering wave vector $\mathbf{q}$ appears in the transition matrix element. Here $\mathbf{q}=\mathbf{k}-\mathbf{k}^{\prime}$, where $\mathbf{k}$ is the wave vector of the incident fast electron and $\mathbf{k}^{\prime}$ the wave vector of the scattered fast electron (corresponding to energies $E$ and $E^{\prime}$ differing by the energy loss). This is the analog of the linear dichroism, which has been observed in XAS. One distinguishing difference between EELS and XAS or similar techniques is that in EELS, one can obtain local atomic scale information by focusing a very small probe of width $\approx 0.1 \mathrm{~nm}$ on a sample in a transmission electron microscope (TEM). This is relevant for studies of nanoscale materials. Modern instruments with field emitters also allow detection of ELNES with an energy resolution of 0.6$0.7 \mathrm{eV}$ on a subnanometer scale and monochromated TEM now can reach $0.1 \mathrm{eV}$ resolution. ${ }^{3}$ However, as we discuss below another important difference between EELS and XAS is due to relativistic corrections.

As for XAS, $a b$ initio calculations are often used to support the interpretation of experimental EELS data. Several approaches exist. One often used approach is based on the independent electron approximation using density-functional theory (DFT) and supercell techniques to treat the core hole, e.g., with the linear augmented plane wave (LAPW) bandstructure code WIEN2K.,5 An alternative is the real-space Green's-function (RSGF) code FEFF, ${ }^{6}$ which does not rely on any assumed periodicity and is applicable to periodic and aperiodic materials alike. In contrast to the DFT-supercell approach, FEFF is based on a real-space, quasiparticle approximation which includes inelastic losses, a screened core hole, and vibrational damping. FEFF was originally designed for XAS but heretofore has been used without modification in EELS studies. However, those calculations implicitly assume an approximate equivalence between dipole-selected EELS (i.e., the long-wavelength limit) and XAS. A review of the application of the FEFF code to EELS calculations is given by Moreno et al. ${ }^{7}$

Although the equivalence between XAS and EELS is often taken for granted, the equivalence is not strictly valid. Recently, for example, it has been recognized ${ }^{8}$ that in anisotropic materials, a relativistic interaction Hamiltonian is essential for accurate calculations of the scattering cross section at relativistic beam energies. The dominant effect is a relativistic contraction of the interaction field, which is consequently anisotropic in the dipole limit. The treatment of this relativistic effect requires a generalization of the FEFF code to account for the anisotropy of the momentum-transfer dependence. This generalization is carried out here and has been implemented in the latest version FEFF9. Note that the dipole-selection rule is an approximation that is generally valid near $\mathbf{q}=0 .{ }^{1}$ This is generally a good approximation for EELS; indeed, for the calculations presented in this paper, nondipole transitions are negligible.

The main purpose of this paper is the development of an $a b$ initio EELS theory and code that is (i) based on a realspace Green's-function approach and (ii) that includes the dominant relativistic effects. This is in contrast to previous work where XAS calculations from FEFF were simply rescaled to fit EELS data. We also discuss the importance of 
instrumental effects (e.g., characteristics of the electron microscope) which are needed to obtain quantitative agreement with modern EELS experiments. The resulting FEFF-based EELS code is broadly applicable and provides a novel, general purpose theoretical tool for EELS calculations, which is complementary to conventional band-structure techniques and applicable over a broad spectral range.

As an illustrative application, we apply the method to calculate the magic angle $\beta_{m}$ in EELS. The magic angle is generally of interest only for anisotropic systems and small collection angles. However, this special angle has a notable relativistic dependence and has been a key issue in the recent developments of relativistic effects in EELS. ${ }^{8}$ The angle is defined as the value of the detector aperture of an electron microscope for which the measured EELS spectrum is independent of the relative orientation of sample and electron beam. Measurements at the magic angle greatly simplify polarized EELS experiments, where the anisotropy of the signal can be an unwelcome complication. Notably the magic angle is material independent, and therefore provides a direct test of the validity of the relativistic theory, unobfuscated by many sources of discrepancy between spectra measured and calculated. Indeed, the recent work that for the first time succeeded in calculating the correct magic angle ${ }^{9}$ has established this angle as a discriminating test that all valid EELS theories and calculations should pass successfully. That is precisely what we aim to do in this paper with the RSGF approach in FEFF. In particular, we present a precise experimental measurement of the magic angle for the $\mathrm{C} K$ edge of graphite, which is compared to FEFF EELS calculations. We show that calculations based on the relativistic generalization of EELS in the FEFF code are more accurate and significantly improve on earlier, nonrelativistic calculations. Although our treatment does not take into account dielectric effects on the magic angle, these effects are negligible for core-level EELS, though they may be important for very low-energy edges. ${ }^{10}$

\section{RELATIVISTIC ELECTRON ENERGY LOSS SPECTRA}

\section{A. Transition tensor formalism}

In this section, we develop a theory of relativistic EELS, in terms of a transition tensor (TT) within the dipole approximation. The EELS signal is conventionally described in terms of the double-differential scattering cross section (DDCS)

$$
\frac{\partial^{2} \sigma(\boldsymbol{q}, E)}{\partial \Omega \partial E},
$$

which is the probability of detecting an electron that has lost energy $E$ and transferred momentum $\mathbf{q}$ by scattering into the solid-angle $d \Omega$. Formally the DDCS can be expressed in terms of the bare Thomson cross section and the relativistic dynamic form factor (DFF) $S(\mathbf{q}, E)$, as in Eq. (2) below. Since the Thomson cross section is sharply peaked at small $q$, it is common practice, and generally a good approximation, to consider only the "dipole transitions" (i.e., small $q$ limit), where the orbital momentum quantum number $l$ of the atomic electron changes by \pm 1 in transitions. Recently it has been shown $^{8,10}$ that in the dipole approximation the relativistic DDSCS for EELS is related to the dynamic form factor $S\left(q^{\prime}, E\right)$ by the relation

$$
\begin{gathered}
\frac{\partial^{2} \sigma(\boldsymbol{q}, E)}{\partial \Omega \partial E}=\left(\frac{\partial \sigma}{\partial \Omega}\right)_{T h} S\left(\boldsymbol{q}^{\prime}, E\right), \\
\left(\frac{\partial \sigma}{\partial \Omega}\right)_{T h}=\frac{4 a_{0}^{-2} \gamma^{2}}{\left[q^{2}-(E / \hbar c)^{2}\right]^{2}} \frac{k^{\prime}}{k}, \\
S\left(\boldsymbol{q}^{\prime}, E\right)=\sum_{i, f}\left|\left\langle i\left|\boldsymbol{q}^{\prime} \cdot \boldsymbol{r}\right| f\right\rangle\right|^{2} \delta\left(E_{f}-E_{i}-E\right) .
\end{gathered}
$$

Here the momentum transfer in the dipole transition element is relativistically contracted and denoted by $q^{\prime}$,

$$
\boldsymbol{q}^{\prime}=\boldsymbol{q}-\beta^{2} q_{z} \hat{\boldsymbol{e}}_{z}
$$

where $\beta=v / c$ and $v$ is the beam velocity. This equation is very similar to the description of XAS in the dipole limit, where the impulse transfer $\mathbf{q}$ plays the role of the polarization vector $\hat{\boldsymbol{\varepsilon}}$ in $\mathrm{X}$-ray scattering matrix elements. However, for relativistic EELS there is an extra q-dependent contribution along the direction of propagation $\hat{\mathbf{e}}_{\mathbf{z}}$.

In general, the DDCS can always be separated into a probe-dependent part containing the $\mathbf{q}$ dependence and a sample-dependent part that is independent of q. Since the theory is bilinear in $\mathbf{q}$, the sample-dependent term transforms as a tensor, i.e.,

$$
\begin{gathered}
S\left(q^{\prime}, E\right)=\sum_{i, j=1}^{3} q_{i}^{\prime} q_{j}^{\prime} \sigma_{i j}(E), \\
\sigma_{i j}(E)=\sum_{i, f}\left\langle i\left|x_{i}\right| f\right\rangle\left\langle f\left|x_{j}\right| i\right\rangle \delta\left(E-E_{i}+E_{f}\right) .
\end{gathered}
$$

Here the TT $\sigma_{i j}(E)$ allows one to describe all angular variations in the dynamic form factor of a sample once and for all, independent of the electron-beam energy. Experimental conditions determine which impulse transfers occur and therefore the weight of each component of the transition tensor that contributes to the total cross section. This can be illustrated by considering the sample to beam orientation of an EELS experiment. Rotation of the sample is equivalent to a rotation of $\mathbf{q}$, thus changing the weights of the $\sigma_{i j}$ components in Eq. (6). The relativistic character of the formalism is also obvious: the field of the beam electron contracts in its propagation direction, resulting in the evaluation of Eq. (6) using a contracted impulse transfer vector, as in Eq. (5), which is denoted by a prime.

Formally, the TT is a real-symmetric tensor with at most six independent components. As such, it can always be diagonalized. However, a priori knowledge of the diagonal representation is only available in symmetric materials, where the principal axes are implied the physical symmetry of the crystal itself and hence one set of coordinates diagonalizes the tensor for all energies. In the general case of a low-symmetry sample or in a situation where a nonsymmetric coordinate system is desirable, the cross terms in Eq. (6) 
are important and cannot be neglected. ${ }^{11,12}$ We give an example of this in Sec. III.

\section{B. Implementation in relativistic EELS code}

Here we discuss how the relativistic theory developed above has been implemented in the FEFF9 code. An EELS experiment usually involves an integration over the DDCS defined in Eq. (2). Typically, the probe has a certain angular width, characterized by the convergence semiangle $\alpha$, allowing a set of incoming plane waves k. Similarly, the detector integrates the signal over a certain range of outgoing beam directions $\mathbf{k}^{\prime}$, characterized by the collection semiangle $\beta$. Both are usually on the order of millirad. Assuming that the incoming beam is monochromatic, the measured signal is then given by

$$
\frac{\partial \sigma(E)}{\partial E}=\int_{\alpha ; \beta} \frac{\partial^{2} \sigma(\boldsymbol{q}, E)}{\partial \Omega \partial E} d^{3} \boldsymbol{q} .
$$

To cast the orientation dependence of the EELS spectrum into a more explicit form, we rewrite Eq. (8) using the TT,

$$
\frac{\partial \sigma(E)}{\partial E}=\sum_{i, j=1}^{3} \sigma_{i j}(E) \int_{\alpha ; \beta} q_{i}^{\prime} q_{j}^{\prime} \frac{4 a_{0}^{-2} \gamma^{2}}{\left[q^{2}-(E / \hbar c)^{2}\right]^{2}} \frac{k^{\prime}}{k} d^{3} \boldsymbol{q} .
$$

Since the TT depends only on the sample system, only integrals over functions of $\mathbf{q}$ need to be calculated to obtain the DDCS. These integrals are approximated by a sum over a finite set of impulse transfer vectors q. Other experimental parameters included in our calculations are the electronbeam energy, the sample to beam orientation, and the position of the EELS detector in the scattering plane. The FEFF9 formalism always includes core-hole broadening appropriate for a given edge, as well as broadening due to final-state self-energy effects. Additional broadening (e.g., to account for experimental resolution) can also be applied.

Our calculations are incorporated as an extension to the $a b$ initio real-space multiple-scattering code FEFF9. The code first calculates the TT, which differs from that in XAS only in the dipole-matrix elements. Next, the code determines the net cross section of Eq. (2) for a given $\mathbf{q}$ from the transition tensor and carries out the integrals over $\mathbf{q}$ as described above. Thus the probe and the sample are treated separately; that is, it is sufficient to calculate the properties of the sample-which are all contained in the TT-only once for the simulation of many experimental situations. All other features of FEFF9 for treating excited states can also be used in EELS calculations. These include the complex GW selfenergy to treat inelastic losses, the time-dependent localdensity approximation formalism to treat local-field effects that correct the independent electron approximation in the calculations, ${ }^{13}$ and Debye-Waller factors to account for disorder and temperature dependent (vibrational) effects.

\section{APPLICATION: C $K$ EDGE OF GRAPHITE}

The strongly anisotropic nature of graphite makes its EELS spectra strongly susceptible to orientational effects

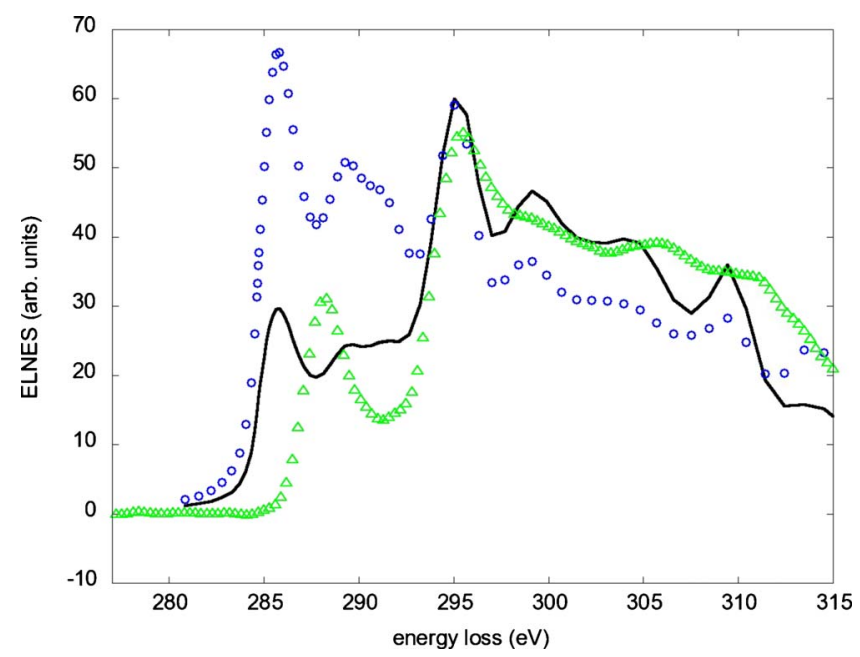

FIG. 1. (Color online) The C $K$ edge of graphite, measured experimentally (solid black line) and calculated relativistically (green triangles) and nonrelativistically (blue circles). Beam at $300 \mathrm{keV}$ along the $c$ axis of the crystal. The convergence angle is $0.2 \mathrm{mrad}$ and the collection angle is $0.68 \mathrm{mrad}$. Experiment is much closer to the relativistic calculation. The $\pi$ to $\sigma$ separation is overestimated in the calculation by several electron volts due to the muffin-tin approximation.

and hence to relativistic contributions to the cross section as discussed in Sec. II. As an illustrative example, we therefore demonstrate our method on the $\mathrm{C} K$ edge of graphite, which has an energy threshold of $285 \mathrm{eV}$. The correspondence of our theoretical spectrum to experimental spectra is on par with other leading $a b$ initio codes but in this paper, we limit ourselves to comparing the calculated spectra to experiment. Figure 1 clearly shows a significant difference between relativistic and nonrelativistic calculation, as a contraction of the electromagnetic field of the beam electron along the direction of propagation reduces the component of the spectrum corresponding to the so-called $\pi$-state transitions, which make up the first part of the spectrum below $290 \mathrm{eV}$ energy loss. As a consequence, the overall spectrum and particularly the ratio of its $\pi$ and $\sigma$ peaks change dramatically. The experiment is seen to be much closer to the relativistic calculation than to the nonrelativistic calculation. There are remaining discrepancies, however, most notably the separation between $\pi$ and $\sigma$ peaks, which is overestimated by several electron volts in our calculations. We believe that this is an artifact of the muffin-tin approximation in FEFF9 and may be remedied by inclusion of the full potential in future developments.

The importance of relativistic corrections depends on the anisotropy of the sample. For isotropic materials, there is no relativistic effect at all, as the relative weights of the components of the spectrum become irrelevant when these components are identical.

Before moving to the study of the magic angle, we briefly discuss the components of the TT and show that calculation of its diagonal components is not generally sufficient to calculate the EELS spectrum. ${ }^{11}$ We work in a Cartesian representation and label the components $i, j$ of the TT as $x, y, z$. We first consider the TT in a symmetrical coordinate system: 


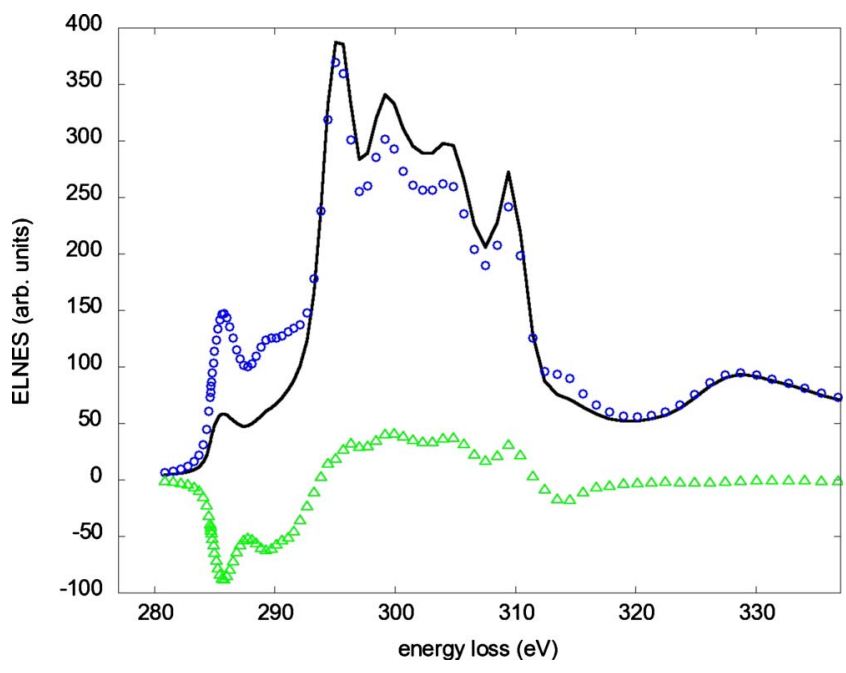

FIG. 2. (Color online) C $K$ edge of graphite calculated relativistically in a symmetric coordinate system (solid black line). Also shown are the diagonal terms $\sigma_{x x}+\sigma_{y y}+\sigma_{z z}$ calculated in an asymmetric coordinate system (see text) (blue circles) and the offdiagonal terms $\sigma_{x y}+\cdots$ (green triangles). In the symmetric coordinate system, the diagonal terms equal the total spectrum and the off-diagonal terms are zero. The total spectrum-sum of diagonal and off-diagonal terms - is the same in both coordinate systems. The beam is perpendicular to the graphene sheets; the beam energy is $300 \mathrm{keV}$; $\alpha=0 \mathrm{mrad}$ (parallel incident beam); and $\beta=10 \mathrm{mrad}$.

its $z$ axis is perpendicular to the graphene sheets of the sample; $x$ and $y$ are in plane. In fully symmetric coordinates, the $\sigma_{z z}$ spectrum contains the so-called $\pi$ transitions or outof-plane transitions. $\sigma_{x x}$ and $\sigma_{y y}$ are identical and contain the so-called $\sigma$ transitions or in-plane transitions. All offdiagonal components are zero, as can be explained by symmetry, i.e., equivalence of $x$ and $-x, y$ and $-y$, and $z$ and $-z$.

We now rotate the $y$ and $z$ axes around the $x$ axis by $35^{\circ}$ and calculate the TT again. $\sigma_{x x}$ is unchanged but $\sigma_{y y}$ and $\sigma_{z z}$ have mixed and are of mixed $\pi$ and $\sigma$ characters. Additionally, the decrease in symmetry allows $y, z$ cross terms to exist. The $x,-x$ symmetry has been preserved, suppressing $x z, z x, x y$, and $y x$ components.

Figure 2 shows the corresponding ELNES spectrum for the $C K$ edge of graphite. In symmetric coordinates, calculation of the diagonal components of $\sigma$ is sufficient. In the rotated system, however, symmetry is broken and the offdiagonal components ( $y z$ and $z y$ in this example) clearly make an important contribution.

As noted in the introduction, the magic angle is defined as that value of the collection angle $\beta_{m}$ for which the EELS spectrum is independent of sample to beam orientation, given a certain convergence angle $\alpha$. In the dipole approximation, one can show that such an angle exists, ${ }^{14}$ at which the integrals in Eq. (9) lose their orientation dependence. The magic angle depends only on beam energy and energy loss but it is approximately constant over the near-edge region. For deep core spectra its value is independent of the material or details of scattering theory. Consequently the magic angle has played a key role in recent developments of EELS theory. ${ }^{8}$ Although many experiments are performed with apertures much larger than the magic angle, it can still be of
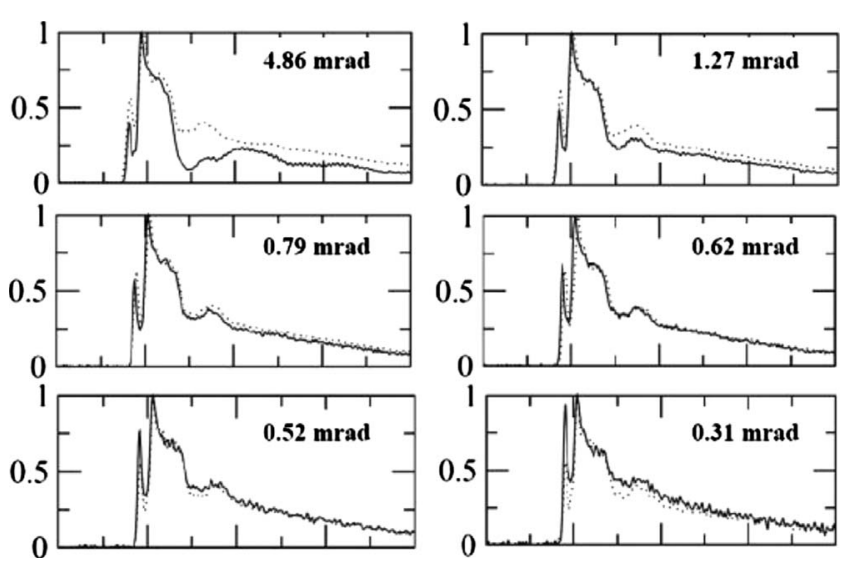

FIG. 3. C $K$ edge of graphite measured at $300 \mathrm{keV}$ beam energy for two orientations and different collection angles. On the horizontal axis is the energy loss (electron volt), ranging from 250 to 450 $\mathrm{eV}$. On the vertical axis, EELS spectra are normalized to 1 . Note that the two orientations overlap between a collection angle of 0.62 and $0.79 \mathrm{mrad}$. The convergence angle is at most $0.2 \mathrm{mrad}$.

practical importance to experimentalists since it allows one to eliminate the complications of orientation dependence. Often this angle is expressed in units of the "characteristic scattering angle" $\theta_{E}$, which is the width of the Lorentzian function that approximates the DFF as a function of scattering angle

$$
\theta_{E}=\frac{E}{E_{0}} \frac{E_{0}+m_{e} c^{2}}{E_{0}+2 m_{e} c^{2}},
$$

where $E_{0}$ is the beam energy, $E$ is the energy loss, and $m_{e}$ is the electron rest mass.

We have performed accurate measurements of the magic angle of graphite. Experimental EELS spectra were recorded on a Jeol 3000F microscope with a GIF 2000 spectrometer. Parallel illumination with convergence angle $\alpha \leq 0.2 \mathrm{mrad}$ was used on a highly oriented pyrolitic graphite sample that was oriented first in 001 zone axis condition and then tilted $30^{\circ}$ away from zone axis condition. The microscope is operated in diffraction mode and the entrance aperture together with the chosen camera length defines the collection angle. A large set of collection angles was taken and selected $\mathrm{C} K$ ELNES spectra are shown in Fig. 3. By definition of the magic angle $\beta_{m}$, the ELNES spectra should not change upon tilting, which occurs experimentally at $\beta_{m}=0.68(9) \mathrm{mrad}$, i.e., between a collection angle of 0.62 and $0.79 \mathrm{mrad}$ and close to $\theta_{E}=0.59 \mathrm{mrad}$.

We now consider theoretical calculations of the magic angle based on our approach, as implemented in FEFF9. We calculate spectra at different sample to beam orientations, which we characterize by a single tilt angle between the electron beam and the crystal $c$ axis. This tilt corresponds to a rotation of $q_{i}^{\prime} q_{j}^{\prime}$ in Eq. (9). We could investigate the rotation invariance of differential cross section, but it is more convenient to choose a more orientation sensitive function of the spectrum, and study it as a function of collection angle at fixed energy loss. At the magic angle, the partial $i=j$ cross sections of Eq. (9) are individually rotation invariant ${ }^{14}$ and 

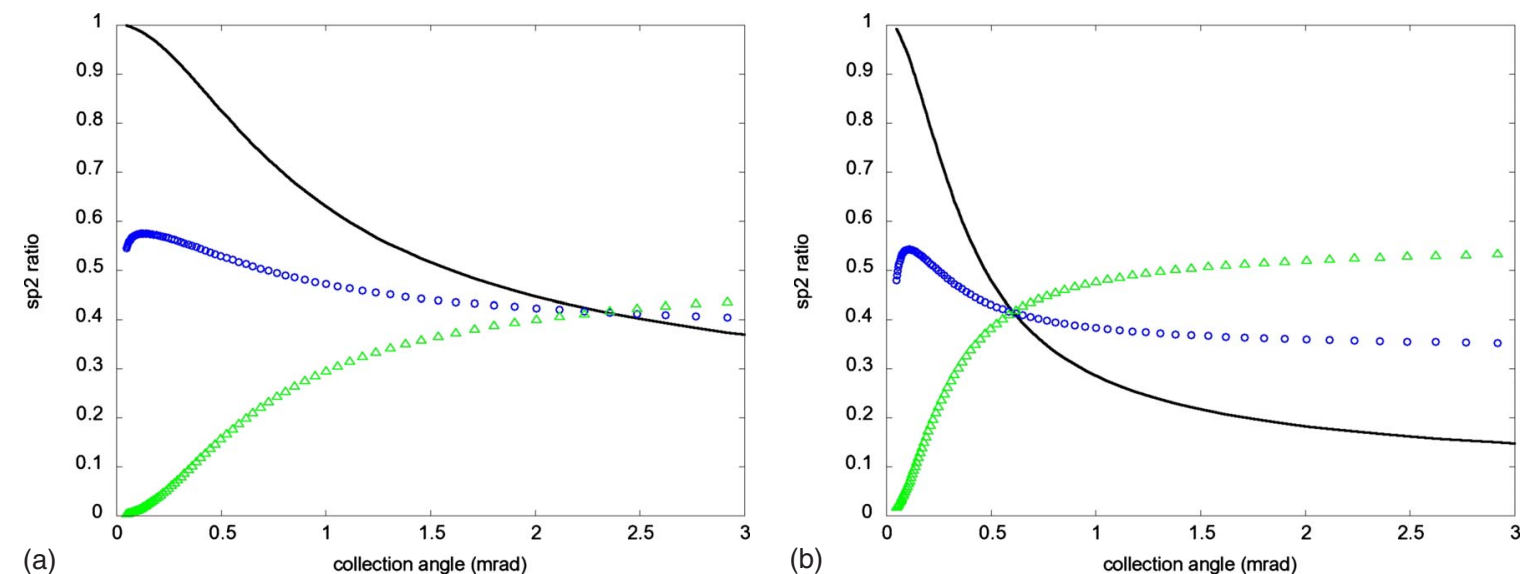

FIG. 4. (Color online) Calculation of the $s p^{2}$ ratio (see text) of the graphite $\mathrm{C} K$ edge at $10 \mathrm{eV}$ above threshold for a $300 \mathrm{keV}$ beam and three sample to beam orientations: $0^{\circ}$ (solid black line), $45^{\circ}$ (blue circles), and $90^{\circ}$ (green triangles). The magic angle is at the intersection of the three curves. The convergence angle is $0 \mathrm{mrad}$ (parallel incident beam). (a) Nonrelativistic calculation shown in left panel and (b) relativistic calculation shown in right panel.

therefore, we may equivalently study the $\pi / \sigma$ ratio of the spectrum. This function is important as it is related to the $s p^{2} / s p^{3}$ ratio which is often used to characterize carbon samples. ${ }^{15}$ In symmetric coordinates, it is given by the $\pi$ term or $i=j=z$ term of Eq. (6), divided by the $\sigma$ term or $i$ $=j=x$ plus $i=j=y$ terms, i.e.,

$$
\left[\frac{\pi}{\sigma}\right]:=\frac{\sigma_{z z}}{\sigma_{x x}+\sigma_{y y}} .
$$

We calculate this quantity at a fixed energy loss of $E$ $=294 \mathrm{eV}$, as a function of collection angle $\beta$ in a nonrelativistic calculation [Fig. 4(a)] and in a relativistic calculation [Fig. 4(b)]. Three different sample to beam orientations are shown in each figure. At the magic angle, the spectrum and its $\pi / \sigma$ ratio are independent of orientation.

The nonrelativistic simulation shown in Fig. 4(a) gives $\beta_{m}=4 \theta_{E}$, a result which has been reported in the literature for many years but is inconsistent with relativistic experiments. A relativistic calculation is shown in Fig. 4(b). We find the magic angle $\beta_{m}=0.60 \mathrm{mrad}$ at zero convergence angle and $\beta_{m}=0.71 \mathrm{mrad}$ at convergence angle $\alpha=0.2 \mathrm{mrad}$, the upper limit of the beam angular width used in the experiment. This theoretical prediction of $0.60-0.73 \mathrm{mrad}$ is in excellent agreement with the experimental measurement of $0.62-0.79$ mrad (Fig. 3). These results also compare well with calculations using the WIEN2K code and with previous work by Hebert. ${ }^{14}$

\section{CONCLUSIONS}

We have presented a relativistic generalization of realspace Green's-function approach that is implemented in the
FEFF9 code for $a b$ initio relativistic calculations of electron energy loss spectra. This approach provides a useful alternative to conventional DFT-based $k$-space calculations of EELS because it is applicable to periodic and aperiodic structured, spans a broad spectral range, and incorporates key many-body effects including core-hole lifetime, final-state self-energy, Debye-Waller factors, and core-hole effects without the need for a supercell. The calculations correctly account for momentum-transfer $\boldsymbol{q}$ dependence and microscope settings such as collection and convergence angle. In contrast to previous FEFF calculations, where EELS spectra were approximated by XAS calculations, the present code is in quantitative agreement with modern EELS experiments with relativistic beam energies. The approach has been tested against recent measurements on the $\mathrm{C} K$ edge of graphite and accurately predicts the observed measured magic angle.

\section{ACKNOWLEDGMENTS}

This work is supported in part by the DOE under Grant No. DE-FG03-97ER45623 (J.J.R.) and facilitated by the DOE Computational Materials Science Network. K. Jorissen gratefully acknowledges financial support by the F.W.O.Vlaanderen as Research Assistant of the Research Foundation-Flanders. We thank L. Allen, Z. Levine, C. Hebert, M. S. Moreno, D. Lamoen, M. Prange, P. Schattschneider, and A. Sorini for comments and suggestions. J. Verbeeck thanks the European Union under the Framework 6 program under a contract for an Integrated Infrastructure Initiative, Reference No. 026019 ESTEEM. 
*Corresponding author; jjr@phys.washington.edu

${ }^{1}$ R. F. Egerton, Electron Energy-Loss Spectroscopy in the Electron Microscope (Plenum Press, New York, 1996).

${ }^{2}$ R. D. Leapman, P. L. Fejes, and J. Silcox, Phys. Rev. B 28, 2361 (1983).

${ }^{3}$ S. Lazar, G. A. Botton, M. Y. Wu, F. D. Tichelaar, and H. W. Zandbergen, Ultramicroscopy 96, 535 (2003).

${ }^{4}$ C. Hebert, Micron 38, 12 (2007).

${ }^{5}$ P. Blaha, K. Schwarz, G. K. H. Madsen, D. Kvasnicka, and J. Luitz, WIEN2k, An Augmented Plane Wave Plus Local Orbitals Program for Calculating Crystal Properties (TU Wien, 2001).

${ }^{6}$ A. L. Ankudinov, B. Ravel, J. J. Rehr, and S. D. Conradson, Phys. Rev. B 58, 7565 (1998).

${ }^{7}$ M. S. Moreno, K. Jorissen, and J. J. Rehr, Micron 38, 1 (2007).

${ }^{8}$ P. Schattschneider, C. Hebert, H. Franco, and B. Jouffrey, Phys.
Rev. B 72, 045142 (2005).

${ }^{9}$ B. Jouffrey, P. Schattschneider, and C. Hebert, Ultramicroscopy 102, 61 (2004).

${ }^{10}$ A. P. Sorini, J. J. Rehr, and Z. H. Levine, Phys. Rev. B 77, 115126 (2008).

${ }^{11}$ M. Nelhiebel, P. H. Louf, P. Schattschneider, P. Blaha, K. Schwarz, and B. Jouffrey, Phys. Rev. B 59, 12807 (1999).

${ }^{12}$ J.-C. Le Bosse, T. Epicier, and H. Chermette, Phys. Rev. B 76, 075127 (2007).

${ }^{13}$ A. L. Ankudinov, Y. Takimoto, and J. J. Rehr, Phys. Rev. B 71, 165110 (2005).

${ }^{14}$ C. Hebert, P. Schattschneider, H. Franco, and B. Jouffrey, Ultramicroscopy 106, 1139 (2006).

${ }^{15}$ J. T. Titantah and D. Lamoen, Phys. Rev. B 70, 075115 (2004). 